\title{
Effect of Finite Ion Temperature and Modulational Instability of Ion-Acoustic Waves in Presence of an Inhomogeneous Plasma
}

\author{
Sikha Bhattacharyya and R. K. Roy Choudhury \\ Electronics Units, Indian Statistical Institute, Calcutta, India
}

Z. Naturforsch. 40 a, 421-424 (1985); received March 7, 1984

\begin{abstract}
Using an extended version of K.B.M. method we have investigated the effect of finite ion temperature on ion-acoustic solitary waves. Modulational instability has been discussed in a frame work of nonlinear Schrödinger equation. Some numerical results are also given.
\end{abstract}

\section{Introduction}

Several authors have made attempts to examine the effect of density gradients on the behaviour of solitons.

Chen and Liu [1] have investigated the non linear propagation in an homogeneous medium in the model of non linear Schrödinger equation. Gell and Gomberoff [2] studied this in the frame work of Kdv model.

The effect of finite ion temperature on ionacoustic solitary waves has been studied by Shivamoggi [3] in a frame work of Korteweg-de Vries model.

Here we have used the extended version of the K.B.M. method (see [4], [5]) to study the effect of finite ion temperature on ion-acoustic solitary waves and derive a non linear Schrödinger equation.

Unlike Shivamoggi we did not have to assume $p_{0}(x)=\left(h_{0}(x)\right)$ from the beginning. We have also discussed the effect of the ion temperature and inhomogeneous terms on the critical values of the wave number for modulational instability.

It was pointed out by Kakutani and Sugimoto [4] that ion acoustic waves with short wave lengths are unstable. More precisely they are unstable for wave numbers $>k_{\mathrm{c}} \simeq 1.471\left(\lambda_{\mathrm{D}}\right)^{-1}$, where $\lambda_{\mathrm{D}}$ is the Debye length.

We find that this value is increased distinctly owing to the presence of finite ion temperature, but the increment is slow for the presence of an inhomogeneous term.

Reprint requests to Prof. Dr. R. K. Roy Choudhury, Electronics Unit, Indian Statistical Institute, 203 Barrackpore Trunk Road, Calcutta 700035 , India.

\section{Mathematical Method}

The fundamental set of equations governing the propagation of non linear ion-acoustic waves for a collision-less plasma composed of a warm-ion fluid and hot isothermal electrons are (see [6] and [7]):

$$
\begin{aligned}
& \frac{\partial n}{\partial t}+\frac{\partial}{\partial x}(n u)=0, \\
& \frac{\partial u}{\partial t}+u \frac{\partial u}{\partial x}=-\frac{\sigma}{n} \frac{\partial p}{\partial x}-\frac{\partial \psi}{\partial x}, \\
& \frac{\partial p}{\partial t}+u \frac{\partial p}{\partial x}+3 p \frac{\partial u}{\partial x}=0, \\
& \frac{\delta^{2} \psi}{\delta x^{2}}=n_{\mathrm{e}}-n, \\
& \frac{1}{n_{\mathrm{e}}} \frac{\delta n_{\mathrm{e}}}{\delta x}=\frac{\partial \psi}{\partial x}=-E,
\end{aligned}
$$

where $n, n_{\mathrm{e}}, u, E$ denote respectively the iondensity, the electron-density, the ion-fluid velocity and the electric field. $\sigma$ is the ratio of ion temperature to electron temperature and $p$ is the ion pressure.

All the variables have been normalized in the following way:

$$
\begin{aligned}
& n=\frac{n_{i}^{\prime}}{N_{0}^{\prime}}, \quad n_{\mathrm{e}}=\frac{n_{\mathrm{e}}^{\prime}}{N_{0}^{\prime}}, \quad u=\frac{u^{\prime}}{\left(K T_{\mathrm{e}}^{\prime} / m_{\mathrm{i}}^{\prime}\right)^{1 / 2}}, \\
& \psi=\frac{\Phi^{\prime}}{K T_{\mathrm{e}}^{\prime} / e}, \quad x=\frac{x^{\prime}}{\left(\frac{K T_{\mathrm{e}}^{\prime}}{4 \pi n_{0}^{\prime} e^{2}}\right)^{1 / 2},} \\
& t=t^{\prime}\left(\frac{4 \pi N_{0}^{\prime} e^{2}}{m_{\mathrm{i}}^{\prime}}\right)^{1 / 2}, \quad \sigma=\frac{T_{\mathrm{i}}^{\prime}}{T_{\mathrm{e}}}, \\
& p=\frac{p^{\prime}}{N_{0}^{\prime} K T_{\mathrm{i}}^{\prime}},
\end{aligned}
$$

0340-4811 / 85 / 0400-0421 \$01.30/0. - Please order a reprint rather than making your own copy. 
where $n^{\prime}$ is the number density, $u^{\prime}$ the flow density $p^{\prime}$ the ion pressure, $T^{\prime}$ the temperature, $\Phi^{\prime}$ the electrostatic potential, and $x^{\prime}$ and $t^{\prime}$ are the space and time coordinates. The primed quantities mean unnormalized variables and the unprimed ones denote the normalized variables. $i$ and $e$ denote respectively the ion and electron and $K$ denotes Boltzmanns constant.

Using (2), (4), (5) we have,

$$
\begin{aligned}
\frac{\partial n}{\partial x} & -\left[\frac{\delta^{3} u}{\delta x \delta t}+\frac{\delta^{2}}{\delta x^{2}}\left(u \frac{\delta u}{\delta x}\right)+\frac{\delta^{2}}{\delta x^{2}}\left(\frac{\sigma}{n} \frac{\delta p}{\delta x}\right)\right] \\
+ & {\left[n \cdot \frac{\delta u}{\delta t}+n u \cdot \frac{\delta u}{\delta x}+\sigma \frac{\delta p}{\delta x}\right] } \\
- & {\left[\frac{\partial}{\partial x}\left(\frac{\sigma}{n} \frac{\partial p}{\partial x}\right)+\frac{\delta^{2} u}{\delta x \delta t}+\frac{1}{2} \frac{\delta^{2}\left(u^{2}\right)}{\delta x^{2}}\right] } \\
. & {\left[\frac{\sigma}{n} \frac{\partial p}{\partial x}+\frac{\delta u}{\delta t}+\frac{1}{2} \frac{\delta}{\delta x}\left(u^{2}\right)\right]=0 }
\end{aligned}
$$

Let us seek the solution of the above equation in the form ( $\varepsilon$ being a small parameter)

$$
\begin{aligned}
& n=n_{0}(x)\left[1+\varepsilon n_{1}+\varepsilon^{2} n_{2}+\varepsilon^{3} n_{3}+\ldots\right], \\
& n=\varepsilon u_{1}+\varepsilon^{2} u_{2}+\varepsilon^{3} u_{3}+\ldots, \\
& p=p_{0}(x)\left[1+\varepsilon p_{1}+\varepsilon^{2} p_{2}+\varepsilon^{3} p_{3}+\ldots\right],
\end{aligned}
$$

where

$$
\begin{aligned}
& \frac{\partial n_{0}}{\partial t}=0, \quad \frac{\partial p_{0}}{\partial t}=0, \quad \frac{\partial p_{0}}{\partial x}=\varepsilon^{2} \alpha_{2}, \\
& \frac{\partial n_{0}}{\partial x}=\varepsilon^{2} \alpha_{1} .
\end{aligned}
$$

If $a, \bar{a}$ denote the complex amplitudes of the plane wave solution of monochromatic emission at zero order, then we have

$$
\begin{aligned}
& u_{1}=a e^{i \psi}+\bar{a} \bar{e}^{i \psi}, \\
& n_{1}=\frac{k}{w}\left(a e^{i \psi}+\bar{a} \bar{e}^{i \psi}\right), \\
& p_{1}=\frac{3 k}{w}\left(a e^{i \psi}+\bar{a} \bar{e}^{i \psi}\right) .
\end{aligned}
$$

The amplitudes $a, \bar{a}$ vary slowly as

$$
\begin{aligned}
& \frac{\partial a}{\partial t}=\varepsilon A_{1}(a, \bar{a})+\varepsilon^{2} A_{2}(a, \bar{a})+\ldots, \\
& \frac{\partial a}{\partial x}=\varepsilon B_{1}(a, \bar{a})+\varepsilon^{2} B_{2}(a, \bar{a})+\ldots
\end{aligned}
$$

together with the complex conjugate of the above relations.

The dispersion relation that follows from the solutions (7) to (9) is as follows:

$$
\left(k^{2}-w^{2}\right)+\frac{3 \sigma p_{0} k^{4}}{n_{0}^{2}}+\frac{3 \sigma p_{0} k^{2}}{n_{0}}=\frac{k^{2} w^{2}}{n_{0}} .
$$

The second set of equations for $0\left(\varepsilon^{2}\right)$ gives the secular free condition

$$
A_{1}+V_{\mathrm{g}} B_{1}=0,
$$

where the group velocity $V_{\mathrm{g}}$ is given by

$$
V_{\mathrm{g}}=\frac{w^{3}}{k^{3}}\left(1+\frac{3 \sigma p_{0} k^{4}}{n_{0}^{2} w^{2}}\right)\left(1-\frac{3 \sigma p_{0} k^{2}}{n_{0} w^{2}}\right) .
$$

Then the order solutions are

$$
\begin{aligned}
& u_{2}=\zeta e^{i \psi}+F_{2} a^{2} e^{2 i \psi}+\mathrm{c} \cdot \mathrm{c}+\gamma^{\prime}, \\
& n_{2}=b_{1} e^{i \psi}+\eta a^{2} e^{2 i \psi}+\mathrm{c} \cdot \mathrm{c}+\gamma, \\
& p_{2}=3 b_{1} e^{i \psi}+F_{3} a^{2} e^{2 i \psi}+\mathrm{c} \cdot \mathrm{c}+\gamma_{p},
\end{aligned}
$$

where

$$
\begin{aligned}
& \xi=\frac{w}{k} b_{1}+B_{1}\left[\frac{i}{k}-\frac{i V_{\mathrm{g}}}{w}\right], \\
& F_{2}=\frac{w}{k} \eta-\frac{k}{w}, \\
& F_{3}=3 \eta+\frac{3 k^{2}}{w^{2}},
\end{aligned}
$$

$$
\begin{aligned}
\eta= & \frac{24 k^{4}+2 k^{2} w^{2}+4 k^{2} n_{0}}{12\left(k^{2} w^{2}-\frac{3 \sigma p_{0} k^{4}}{n_{0}}\right)} \\
& +\frac{\sigma p_{0} k^{4}\left[\frac{24 k^{2}}{w^{2} n_{0}}+\frac{18 \sigma p_{0}^{2} k}{n_{0}^{2} w^{2}}-\frac{12}{n_{0}}+\frac{12}{w^{2}}\right]}{12\left(k^{2} w^{2}-\frac{3 \sigma p_{0} k^{4}}{n_{0}}\right)},
\end{aligned}
$$

and $b_{1}, \bar{b}_{1}$ (complex) and $\gamma, \gamma^{\prime}, \gamma_{p}$ (real) are constants independent of $w$ but dependent on $a, \bar{a}$. They are determined from the non secular conditions at higher orders. c. c. denotes complex conjugate. 
Applying the non secularity conditions at $0\left(\varepsilon^{3}\right)$ we obtain

$$
\begin{aligned}
& \gamma^{\prime}=\frac{\left(\frac{2 k n_{0}}{w}+w^{2} V_{\mathrm{g}}\right)+\frac{6 \sigma p_{0} k}{w}\left(1+\frac{k}{w}-\frac{k w V_{\mathrm{g}}}{n_{0}}+\frac{3}{2} \frac{k^{2} \sigma p_{0} V_{\mathrm{g}}}{n_{0}^{2} w}\right)}{\left(V_{\mathrm{g}}^{2}-1-\frac{3 \sigma p_{0}}{n_{0}}\right) n_{0}} a+\lambda_{1}, \\
& \gamma=\frac{\left(\frac{2 k V_{\mathrm{g}} n_{0}}{w}+w^{2}\right)+\frac{6 \sigma p_{0} k}{w V_{\mathrm{g}}}\left(\frac{k}{w}-\frac{k w V_{\mathrm{g}}}{n_{0}}+\frac{3}{2} \frac{p_{0} \omega k^{3} V_{\mathrm{g}}}{n_{0}^{2} w}\right)}{\left(V_{\mathrm{g}}^{2}-1-3 \frac{\sigma p_{0}}{n_{0}}\right) n_{0}} a+\lambda_{2},
\end{aligned}
$$

$$
\begin{aligned}
\gamma_{p}= & {\left[\left(\frac{6 k n_{0} V_{\mathrm{g}}}{w}+3 w^{2}+\frac{6 k^{2}}{w^{2}}\left(V_{\mathrm{g}}^{2}-1\right)+\frac{18 \sigma p_{0} k}{w V_{\mathrm{g}}}\left(\frac{k}{w}-\frac{k V_{\mathrm{g}}}{w}-\frac{k V_{\mathrm{g}} w}{n_{0}}\right.\right.\right.} \\
& \left.\left.+\frac{3}{2} k^{3} \frac{\sigma p_{0} V_{\mathrm{g}}}{n_{0}^{2} w}\right)\right] a \bar{a} /\left[\left(V_{\mathrm{g}}^{2}-1-\frac{3 \sigma p_{0}}{n_{0}}\right) n_{0}\right]+\lambda_{3},
\end{aligned}
$$

where $\lambda_{1}, \lambda_{2}, \lambda_{3}$ are arbitrary constants. When $\sigma=0, \gamma^{\prime}, \gamma$ conform to the parameters $c_{1}, c_{2}$ (see (30)) of [4]).

Before we derive the nonlinear Schrödinger equation we find that elimination of the resonant terms gives

$$
i\left(A_{2}+V_{\mathrm{g}} B_{2}\right)+P\left[B_{1} \frac{\partial B_{1}}{\partial a}+\bar{B}_{1} \frac{\partial B_{1}}{\partial a}\right]=Q a^{2} \bar{a}+R a,
$$

where

$$
\begin{aligned}
P= & {\left[-\frac{3 w^{3}}{2 n_{0} k^{4}}-\frac{3}{2 n_{0} k}\left(C_{V}-\frac{3 \sigma p_{0} k}{n_{0}}\right)\right.} \\
& \left.+\frac{1}{2 k^{2}}\left(1+k^{2} / n_{0}\right)\left(1 / k-V_{\mathrm{g}} / w\right)\left(C_{V}+\frac{3 \sigma p_{0} w}{n_{0} k}\right)\right]\left[\left(w^{2}-\frac{3 \sigma p_{0} k^{2}}{n_{0}}\right)\right], \\
Q= & {\left[\left\{\frac{3}{2} \frac{w}{n_{0}}+\frac{w}{k^{2}}-\frac{w^{3}}{k^{2} n_{0}}+\frac{\sigma p_{0}}{2 n_{0}^{2} w}\left(3 k^{2}+12 w^{2}-6 n_{0}-\frac{18 \sigma p_{0} k^{2}}{n_{0}}\right)\right\} \eta\right.} \\
& +\left\{\left(\frac{1}{2 w}-\frac{k^{2}}{n_{0} w}+\frac{3}{2} \frac{w}{n_{0}}\right)-\frac{\sigma p_{0} k^{2}}{2 n_{0}^{2} w^{2}}\left(21 k^{2}+\frac{9 \sigma p_{0} k^{2}}{n_{0}}+6 w^{3}+15 n_{0}\right)\right\} \\
& +\gamma_{\mathrm{s}}^{\prime}\left\{\frac{1}{k}\left(1+k^{2} / n_{0}\right)+\frac{3}{2} \frac{\sigma p_{0} k}{w^{2} n_{0}^{2}}\left(k^{2}+n_{0}\right)(1-w)\right\} \\
& \left.+\gamma_{\mathrm{s}}\left\{\frac{w}{2 n_{0}}-\frac{3}{2} \frac{\sigma p_{0}}{n_{0}^{2} w}\left(n_{0}+2 k^{2}\right)\right\}+\frac{3 \sigma k_{0}}{2 n_{0}^{2} w} \gamma_{\mathrm{ps}}\left(k^{2}-n_{0}\right)\right]\left[w^{2}-\frac{3 \sigma p_{0} k^{2}}{n_{0}}\right], \\
R= & -\alpha_{1}\left(\frac{1}{n_{0} k w}+\frac{3 \sigma p_{0} k}{n_{0}^{3} w}\right) i-\alpha_{2} i\left(\frac{9 \sigma k}{2 n_{0} w}+\frac{2 \sigma}{n_{0} k w}\right) \\
& \left.+\frac{3}{2} \frac{\sigma^{2} p_{0} k}{n_{0}^{3} w}-\frac{\sigma w}{2 n_{0}^{2} k}\right)+\left\{\frac{k}{w^{2}}+\frac{3 \sigma p_{0} k}{2 n_{0} w}\left(\frac{3}{w}-1\right)\left(\frac{k^{2}}{n_{0}}+1\right)\right\} \lambda_{1} \\
& \left.+\left\{\frac{w}{2 n_{0}}-\frac{3 \sigma p_{0}}{2 n_{0}^{2} w}\left(n_{0}+2 k^{2}\right)\right\} \lambda_{2}+\frac{3}{2} \sigma p_{0}\left(\frac{k^{2}}{n_{0}^{2} w}+\frac{1}{n_{0} w}\right) \lambda_{3}\right]\left[\left(w^{2}-\frac{3 \sigma p_{0} k^{2}}{n_{0}}\right)\right],
\end{aligned}
$$


here $\gamma_{s}^{\prime}, \gamma_{s}, \gamma_{\mathrm{ps}}$ are the coefficients of $a \bar{a}$ in $\gamma^{\prime}, \gamma$ and $\gamma_{\mathrm{p}}$ respectively, and

$$
C_{V}=\left(\frac{-3 \sigma p_{0} w}{n_{0} k}+\frac{3 \sigma p_{0} k w}{n_{0}^{2}}-\frac{9 \sigma^{2} p_{0}^{2} k^{3}}{n_{0}^{3} w}\right) .
$$

Introducing the coordinate transformation defined as

$$
\begin{aligned}
& \xi=\frac{1}{\varepsilon}\left(x_{2}-V_{\mathrm{g}} t_{2}\right)=\varepsilon\left(x-V_{\mathrm{g}} t\right), \\
& \tau=t_{2}=\varepsilon^{2} t,
\end{aligned}
$$

(25) can be reduced to the nonlinear Schrödinger equation

$$
i \frac{\partial a}{\partial \tau}+P \frac{\delta^{2} a}{\delta \xi^{2}}=Q\left|a^{2}\right| a+R a .
$$

When $\sigma=\alpha_{2}=0, \alpha_{1}=0, P, Q, R$ conform to the form given by Kakutani and Sugimoto [4].

\section{Discussion and Conclusion}

It was shown by Tanuiti and Yajima [8] and also Hasimoto and Ono [9] that the plane wave solution of the nonlinear Schrödinger equation is modulationally unstable if $P Q<0$.

[1] H. H. Chen and C. S. Liu, Phys. Fluids 21, 377 (1978).

[2] Y. Gell and L. Gomberoff, Phys. Lett. A, 60, 125 (1977).

[3] Bhimsen K. Shivamoggi, Canad. J. Phys. 59, 719 (1981).

[4] T. Kakutani and N. Sugimoto, Phys. Fluids 17, 1617 (1975).

[5] I. R. Durrani, G. Murtaza, and H. U. Rahman, Canad. J. Phys., 57, 642 (1979).
Now, if $k_{\mathrm{c}}$ denotes the value such that $P Q \gtrless 0$ according as $k \gtrless k_{\mathrm{c}}$ then $k_{\mathrm{c}}$ is called the critical wave number.

To see the effect of finite ion temperature and inhomogeneity we take $n_{0}(x), p_{0}(x)$ of the form $p_{0}(x)=n_{0}(x)=n_{0}(1+a x)$, where $a$ is a small num-

\begin{tabular}{|c|c|c|c|}
\hline & 0 & 0.001 & 0.01 \\
\hline 0 & 1.471 & 1.605 & 1.650 \\
\hline 0.02 & 1.485 & 1.603 & 1.672 \\
\hline 0.025 & 1.490 & 1.627 & 1.678 \\
\hline 0.033 & 1.50 & 1.635 & 1.686 \\
\hline
\end{tabular}
ber given by $a=1 / L$ and numerically evaluate $k_{\mathrm{c}}$ for some selected values of $a$ :

Values of $k_{\mathrm{c}}$.

Our results for $\sigma=0, a \neq 0$ differ from those of Durrani et al. [5]. But it must be mentioned that their results do not conform to those obtained by Kakutani and Sugimoto in the limit $\lambda \rightarrow 1$. Also it seems that their values for $k_{\mathrm{c}}$ do not conform to their own formula for $Q$ as given in [5].

The above table shows that the values of $k_{\mathrm{c}}$ increase as $\sigma$ increases. The same holds for the inhomogeneity parameter $a$, but in the latter case the rate of increase is slow and proportional to $\sqrt{n_{0}}$.

[6] D. A. Tidman and H. M. Stainer, Phys. Fluids 8, 345 (1965).

[7] A. H. Nayfeh, Phys. Fluids 8, 1896 (1965).

[8] T. Taniuti and N. Yajima, J. Math. Phys. 10, 1369 (1969).

[9] H. Hasimoto and H. Ono, J. Phys. Soc. Japan 33, 805 (1972). 